\title{
El currículo en contextos de estudiantes migrantes: Las complejidades del desarrollo curricular desde la perspectiva de los docentes de aula
}

\author{
Curriculum in contexts of migrant students: The complexities of curriculum development \\ from the perspective of classroom teachers \\ O currículo no contexto dos alunos migrantes: As complexidades do desenvolvimento
curricular a partir da perspectiva dos professores em sala de aula
}

\author{
Arnaldo Hernández Yulcerán \\ Magíster en Educación mención en Currículo Educacional. Licenciado en Educación \\ Profesor de Educación General Básica \\ Telf.: (41)22956219. Correo electrónico: arnaldohy@gmail.com
}

\begin{abstract}
RESUMEN
Las estadísticas actuales indican que Chile es uno de los principales destinos migratorios dentro de la región. El aumento de la población migrante ha generado una transformación en la cultura educacional del país, ampliando la diversidad que comúnmente debía atender la escuela. Sin embargo, las investigaciones que han indagado sobre los procesos de integración de niños y niñas migrantes, revelan la presencia de conductas discriminatorias y racistas por parte de la comunidad educativa. Por otro lado, se vislumbra que el currículo implementado en estos establecimientos es descontextualizado. Además, a nivel gubernamental existe una ausencia de políticas educativas, que fomenten propuestas curriculares interculturales para abordar la diversidad étnica-cultural de la migración. Por lo tanto, el objetivo de esta investigación es "describir el desarrollo curricular de docentes que trabajan en dos establecimientos educativos municipales con estudiantes migrantes de la comuna de Santiago".
\end{abstract}

Palabras clave: migración, diversidad cultural, educación intercultural, currículum, desarrollo curricular.

ABSTRACT
Current statistics indicate that Chile is one of the main migration destinations within the region. The increase in
the migrant population has generated a transformation in the educational culture of the country, expanding the
diversity that was commonly attended at the school. However, studies investigating the processes of integration of
migrant children reveal the presence of discriminatory and racist behavior by the educational community. On the
other hand it can be seen that the curriculum implemented in these establishments is decontextualized. In addition,
at the government level there is a lack of educational policies that foster intercultural curriculum proposals to
address the ethnic-culture diversity of migration. Therefore, the objective of this research is to "describe the
curricular development of teachers working in two urban schools with migrant students in the district of Santiago".

Key words: migration, cultural diversity, intercultural education, curriculum, curriculum development. 


\section{INTRODUCCIÓN}

Desde la década de los noventa en adelante el país experimentó un aumento considerable en la recepción de población extranjera, tendencia que de acuerdo a los resultados del Censo año 2002 demostró que "existe un predominio de inmigración argentina, pero también evidenció que son los inmigrantes peruanos, bolivianos, ecuatorianos y colombianos los que más aumentaron respecto del decenio anterior" (Instituto Nacional de Estadísticas [INE], 2003). Además, los datos indican que "la población peruana y ecuatoriana, por ejemplo, creció casi un 400\% entre 1992 y 2002". (Soffia, Cano y Martínez, 2009: 15). La medición censal de ese año determinó que el total de personas migrantes era de 184464 , de las cuales casi el $70 \%$ pertenecían a países del Cono Sur, como Argentina, Perú, Ecuador y Colombia.

La cantidad de extranjeros residentes según los resultados del censo año 2012 (INE, 2014) es de 327074, pero según estimaciones realizadas por el Departamento de Asuntos Económicos y Sociales de las Naciones Unidas (2013) la cifra ascendería a 398251 migrantes. A diferencia de lo que sucede con la población total de migrantes, existe información más fidedigna en cuanto a su distribución en el territorio nacional pudiéndose identificar cuatro regiones del país que configuran los puntos claves de la ruta migratoria. Estas ciudades son: Santiago (Región Metropolitana), Arica (Región de Arica-Parinacota), Iquique (Región de Tarapacá) y Antofagasta (Región de Antofagasta) (Torres ,2011).

Más allá de las cifras que en nuestro caso todavía no constituyen un problema de envergadura nacional, el punto crítico lo representan las precarias condiciones de vida en las cuales vive la población migrante. Esta situación ha sido advertida tanto por organismos internacionales como nacionales, los que señalan la vulneración de derechos fundamentales hacia los migrantes. El derecho a la educación, la salud, la vivienda, al trabajo digno, a tener nacionalidad, entre otros, son frecuentemente transgredidos, incluso a pesar de existir algunos mecanismos que debieran resguardar su ejercicio (Martínez, 2003; Stefoni, 2011; Torres, 2011).

\section{EDUCACIÓN DE LOS NIÑOS Y JÓVENES MIGRANTES EN CHILE}

A nivel latinoamericano, la OEA (2011) realiza un diagnóstico sobre la educación de niños y jóvenes migrantes. Los resultados demuestran que todavía este tema no logra convertirse en un campo de investigación propio. Habitualmente, la investigación académica se ha enfocado más en el tráfico, la explotación, la reunificación familiar o en la integración general de la infancia migrante, donde entra la educación como tema secundario. Además de la ausencia de estudios en la región, se detectó que el fenómeno migratorio se encuentra muchas veces incluido dentro de políticas públicas sobre diversidad o minorías étnicas de un determinado territorio. El informe señala dentro de sus conclusiones que:

La población de niños y jóvenes migrantes presenta necesidades específicas que deben ser tomadas en cuenta a la hora de pensar en políticas públicas, como el idioma, la integración cultural, el desarraigo con el país de origen, la separación de las familias y su reencuentro, la interrupción en los estudios y el reconocimiento de los certificados educativos.

El currículo escolar debe contemplar las diferencias culturales, de lengua y de identidad de los sujetos. 
Por lo tanto, la educación de la infancia migrante se transforma en un gran desafío que la escuela debe afrontar, ya que la institucionalidad educativa no está preparada para integrar a los niños y jóvenes en un enfoque de derechos humanos donde se respete su identidad y cultura de origen. En este aspecto, el currículo representa un punto de tensión que limita el diálogo intercultural entre los distintos actores del proceso educativo.

Respecto al acceso a la educación, en Chile se han hecho algunas modificaciones administrativas y jurídicas para resguardar los derechos humanos de los niños migrantes. En esa dirección, el MINEDUC ha emitido algunos Instructivos durante el 2001, 2003 y 2005 con la finalidad de garantizar el ingreso de niños y jóvenes migrantes a los centros escolares con independencia de su situación migratoria o la de sus padres. El último cambio fue la creación de la "matrícula provisoria" que autoriza el pago de la subvención escolar a los sostenedores mientras se legalizan los certificados académicos de los estudiantes extranjeros.

Estas medidas representan un avance que garantiza el derecho a la educación, aunque todavía se producen algunas dificultades en establecimientos educativos. Esto fue constatado por el equipo del proyecto "Ciudadanía y protección de los derechos humanos de la población inmigrante en Chile" (Torres ,2011), que registró denuncias tanto en Santiago como en ciudades de la zona norte, que demuestran que se sigue obstaculizando la matrícula a los estudiantes extranjeros cuyos padres se encuentran en situación migratoria irregular. De esta forma se desconoce la normativa vigente que garantiza el acceso a la educación de todo niño o joven sin distinción de nacionalidad, sexo, género, cultura, religión, etnia, situación socioeconómica o migratoria, todos aspectos contemplados en los tratados internacionales ratificados por Chile y cuyo incumplimiento constituye una vulneración manifiesta a las obligaciones contraídas por el Estado chileno.

Aunque se detectó que los estudiantes migrantes todavía poseen dificultades para acceder a la educación formal, la encuesta CASEN del 2006 (Cit. en Soffia et al., 2009) en uno de sus ítems sobre la cobertura del sistema educativo chileno de los migrantes en edad escolar, muestra que casi la totalidad de los que tienen entre 6 y 17 años asiste actualmente a algún establecimiento educacional $(95,4 \%)$, cifra que es prácticamente igual al promedio de Chile (96,5\%). Éste es un antecedente a considerar cuando las estadísticas por distribución geográfica señalan que la Región Metropolitana es el lugar con mayor concentración migrante en el país, específicamente de ciudadanos peruanos.

Una de las consecuencias producidas por la concentración de población migrante es que durante este último tiempo en las escuelas municipales de la Región Metropolitana se ha observado un aumento sostenido en la matrícula de estudiantes extranjeros. De acuerdo a la base de datos del MINEDUC por concepto de matrícula año 2013, existen en la comuna de Santiago varios establecimientos que se caracterizan por tener a más del $50 \%$ del alumnado de origen migrante ${ }^{1}$. Dentro de los casos más representativos y que han sido estudiados destacan la escuela República de Alemania, República de Israel, República de Panamá y República de Colombia.

La encuesta realizada por la UNICEF en el 2004 sobre prejuicios a alumnos de otras nacionalidades, llevada a cabo en las ciudades de Iquique, Santiago y Temuco, mostró que el $46 \%$ de las niñas y niños adolescentes chilenos consideraba que una o más nacionalidades

El detalle de la matrícula año 2013 de establecimientos ubicados en la comuna de Santiago Centro fue solicitado al MINEDUC bajo la ley de transparencia. 
eran inferiores que la chilena. Las nacionalidades vistas más negativamente fueron la peruana (32\%), boliviana (30\%) y argentina (16\%), las que corresponden precisamente a las tres nacionalidades de países de origen con mayor presencia en Chile (www. educarchile.cl). Por otro lado, la "Primera Consulta Ciudadana sobre la Discriminación en Chile" (Ministerio Secretaría General de Gobierno, 2013) complementa la información obtenida por UNICEF, indicando que la tercera causa más recurrente por la cual se discrimina en el país corresponde precisamente a la "nacionalidad". Además, según los consultados, los establecimientos educacionales estarían dentro de los espacios donde se ejerce generalmente la discriminación, obteniendo el segundo lugar después de plazas y los lugares públicos. Los datos resultan preocupantes, pues existe evidencia suficiente que demuestra la presencia de estereotipos y prejuicios que se están reproduciendo al interior de los establecimientos educativos.

Los resultados de las investigaciones realizadas en escuelas de la comuna de Santiago revelan que la integración de los estudiantes migrantes, particularmente de niños peruanos, (Pavez, 2013; Tijoux, 2013) es compleja porque deben afrontar conductas discriminatorias y racistas por parte de la comunidad educativa. Coincidiendo con este diagnóstico, Poblete (2006) y Aranda (2011) señalan dos aspectos claves a la hora de pensar en una política curricular que permita abordar la diversidad cultural. Por un lado la falta de preparación de los docentes para afrontar el conflicto cultural debido a una formación inicial homogénea y la implementación de un currículo descontextualizado. En segundo lugar, la necesidad de contar con una institucionalidad sólida por parte del ministerio de educación y los municipios, que fomente y apoye proyectos de innovación curricular. En este escenario el equipo del proyecto "Ciudadanía y protección de los derechos humanos de la población inmigrante en Chile" (Torres, 2011), recomienda y considera esencial incorporar en el currículo educacional contenidos a favor de la interculturalidad y la integración en el marco de los derechos humanos.

Frente a la necesidad de implementar programas de Educación Intercultural que permitan avanzar en la eliminación de prejuicios y estereotipos hacia los estudiantes migrantes, la única experiencia concreta que se conoce en la Región Metropolitana es la elaboración de un plan y programa de estudio para los $7^{\circ}$ básicos de tres escuelas con alto alumnado migrante en la comuna de Santiago Centro (Escuela República de Alemania, Escuela República Israel y Escuela República Panamá), denominado "Historia, geografía y ciencias sociales de Suramérica”. En palabras de Hector Pezoa, Vicepresidente de la ONG Secretaría Ciudadana de Migrantes en Chile, la elaboración del programa curricular se fundamenta porque "las mallas curriculares actuales están diseñadas con una orientación de las ciencias sociales dirigidas fundamentalmente a la nacionalidad chilena, y lo que se aspira con esta nueva programación es abrir los horizontes de los educandos hacia una visión americanista; lográndose que los niños no tan solo tengan esa perspectiva amplia, sino que puedan practicar la convivencia con sus compañeros de otras nacionalidades"'.

De acuerdo a lo planificado, el programa de estudio se comenzó a implementar de modo experimental a partir del segundo semestre del 2009, sin embargo, al consultar

\footnotetext{
Información obtenida en una entrevista realizada a Hector Pezoa Vicepresidente de la ONG Secretaría Ciudadana de Migrantes en Chile sobre el programa de estudio "Historia, geografía y ciencias sociales de Suramérica", publicada por la Dirección de Bibliotecas, Archivos y Museos (DIBAM). En http://www.contenidoslocales.cl/comunidad/2851/innovacioncurricular-para-colegios-con-alto-alumnado-inmigrante.
} 
directamente con los profesores encargados de desarrollar la nueva asignatura se verificó que la escuela República de Israel solo participó en la fase inicial de la programación del proyecto, a diferencia de la escuela República de Panamá donde el programa logró implementarse hasta el año 2013. Actualmente, el programa curricular solo se realiza en la escuela República de Alemania. En este contexto llama la atención que en otras comunas donde existe también una alta concentración de familias migrantes como Recoleta, Estación Central e Independencia todavía no se han generado propuestas de Educación Intercultural o innovaciones curriculares que reconozcan la diversidad cultural del alumnado migrante.

Considerando la magnitud del fenómeno que está impactando al sistema educativo nacional y la poca investigación existente en el área de educación, es necesario generar información que oriente la futura elaboración de políticas educativas que aborden a nivel curricular la situación de los estudiantes de origen migrante.

\section{DISEÑO METODOLÓGICO}

La aproximación al objeto de estudio fue realizada desde el paradigma cualitativo, a través de un estudio de casos múltiples. Las técnicas de recolección de información empleadas fueron la entrevista en profundidad y la observación no participante, pues uno de los objetivos principales del estudio era recoger información de alto contenido. El uso de los métodos escogidos se fundamenta por obtener información de manera inmediata, personal y a través del contacto directo con la realidad.

Los establecimientos educativos que formaron parte de este estudio son la escuela República de Colombia y la escuela Fernando Alessandri Rodríguez. Ambas son de dependencia municipal, pertenecen a la comuna de Santiago Centro y poseen una numerosa cantidad de estudiantes migrantes sudamericanos. Los sujetos informantes de cada establecimiento fueron profesores de Educación Básica que poseen jefaturas desde $1^{\circ}$ a $4^{\circ}$ año básico. Durante el trabajo de campo se realizaron 12 entrevistas en profundidad y 11 observaciones no participantes, ya que uno de los docentes no autorizó la observación de clases.

Respecto a la observación de clases es importante señalar que estas se realizarían, de preferencia, en las asignaturas de Lenguaje y Comunicación, Historia, geografía y ciencias sociales y Ciencias Naturales. La relevancia de que las observaciones se realizaran en estas asignaturas tiene estrecha relación con los supuestos enunciados en la investigación, pues se cree que en estas áreas del saber se estarían produciendo mayores complejidades curriculares por tratarse contenidos más locales. Finalmente y en concordancia con el horario y preferencia de los profesores, las observaciones de clases se efectuaron solo en las asignaturas de Lenguaje y comunicación e Historia, geografía y ciencias sociales.

Una vez concluido el trabajo de campo se procedió al análisis de las entrevistas en profundidad y de las pautas empleadas en la observación no participante (escala de Likert y notas de campo adicionales). El análisis de contenido se realizó a través de categorías y subcategorías que surgieron de los objetivos específicos como del marco teórico existente. También es importante señalar que durante las observaciones de clases se incorporó una subcategoría emergente. A continuación se muestra un cuadro con las categorías y subcategorías y su definición: 
Cuadro 1. Categorías y subcategorías de análisis

\begin{tabular}{|c|c|}
\hline CATEGORÍAS & SUBCATEGORÍAS \\
\hline $\begin{array}{l}\text { Interacciones Sociales: Conjunto } \\
\text { de relaciones sociales que se ori- } \\
\text { ginan en el aula entre los distin- } \\
\text { tos sujetos participantes (docen- } \\
\text { te, estudiantes nacionales y mi- } \\
\text { grantes). Específicamente, en } \\
\text { este estudio por relaciones socia- } \\
\text { les se entenderán las representa- } \\
\text { ciones sociales o percepciones } \\
\text { culturales que posea el docente, } \\
\text { el trato que se genera entre todos } \\
\text { los actores educativos (discrimi- } \\
\text { nación o aceptación del otro) y } \\
\text { por último, la participación de } \\
\text { los estudiantes migrantes durante } \\
\text { el desarrollo de las clases. }\end{array}$ & $\begin{array}{l}\text { Representaciones Sociales: Conjunto de conceptos, declara- } \\
\text { ciones y explicaciones sobre los estudiantes migrantes. Ori- } \\
\text { ginadas en el quehacer pedagógico cotidiano de los docentes, } \\
\text { en el curso de las comunicaciones interindividuales, que se } \\
\text { producen al interior de la comunidad educativa. } \\
\text { Inclusión Educativa: Características del proceso de integra- } \\
\text { ción de los estudiantes migrantes en sus respectivos niveles } \\
\text { educativos. En primer lugar de las conductas en general rea- } \\
\text { lizadas por los docentes y estudiantes nacionales hacia los } \\
\text { niños migrantes, y, por otro lado, al protagonismo de los es- } \\
\text { tudiantes migrantes en las actividades de aprendizaje. } \\
\text { Espacio y distribución (Subcategoría Emergente): El espacio } \\
\text { se refiere a los lugares físicos al interior del aula destinados } \\
\text { para representar aspectos culturales de los distintos países; y } \\
\text { la distribución corresponde a la asignación de los puestos que } \\
\text { ocupan los estudiantes migrantes en clases. }\end{array}$ \\
\hline $\begin{array}{l}\text { Desarrollo Curricular: Proceso a } \\
\text { través del cual el currículo co- } \\
\text { mienza a experimentar interpre- } \\
\text { taciones, transformaciones y re- } \\
\text { contextualizaciones producto de } \\
\text { la mediación entre la cultura de } \\
\text { la escuela y el cuerpo docente. } \\
\text { Esta etapa ocurre después de la } \\
\text { implementación de la propuesta } \\
\text { curricular oficial y marca una } \\
\text { distinción entre el currículo pres- } \\
\text { crito y aplicado. }\end{array}$ & $\begin{array}{l}\text { Contextualización Curricular: Interpretación del currículo } \\
\text { oficial desde la experiencia profesional, para poder articular } \\
\text { los contenidos declarados en el marco curricular con los sa- } \\
\text { beres culturales de los estudiantes migrantes. Las adecuacio- } \\
\text { nes curriculares pueden surgir previamente por un proceso de } \\
\text { planificación curricular o de manera emergente durante el } \\
\text { desarrollo de las clases. } \\
\text { Significación Curricular: Evaluación personal que realiza el } \\
\text { docente de los lineamientos sobre diversidad cultural presen- } \\
\text { tes en la propuesta curricular oficial. } \\
\text { Implementación curricular: Implementación de actividades } \\
\text { de enseñanza-aprendizaje para abordar la diversidad cultural } \\
\text { de los estudiantes migrantes, que incluyan temáticas trans- } \\
\text { versales comunes, como por ejemplo comidas típicas, vesti- } \\
\text { menta, modismos, recursos naturales y otros. } \\
\text { Materiales curriculares: Instrumentos curriculares y material } \\
\text { didáctico empleado por los docentes que se desempeñan con } \\
\text { estudiantes migrantes. Aquí se incluyen los insumos curricu- } \\
\text { lares dispuestos por el MINEDUC u otros complementarios } \\
\text { de elaboración personal. }\end{array}$ \\
\hline
\end{tabular}




\section{RESULTADOS Y DISCUSIÓN}

\subsection{INTERACCIONES SOCIALES: EL PRIMER PASO PARA EL RECONOCIMIENTO DEL OTRO, DE} ESE OTRO, EL ESTUDIANTE MIGRANTE

Los profesores reconocen que con la llegada de estudiantes migrantes se han producido intercambios culturales entre los distintos actores que forman parte del proceso educativo. Los intercambios más recurrentes tienen relación con temas como las comidas, bailes, vestuario, artesanías y lugares geográficos. También se han generado algunas instancias para compartir experiencias familiares y personales sobre el proyecto migratorio. Como las causas que motivaron a sus padres a migrar, la ocupación laboral de estos y la recepción que han tenido en Chile. Las intervenciones realizadas por los estudiantes migrantes son apreciadas por sus pares chilenos. Producto de esos episodios se han producido comparaciones entre la cultura chilena y las otras culturas. Las que han sido gatilladas por los docentes a través de preguntas o de manera espontánea por los niños y niñas migrantes al referirse a un determinado tema. Por ejemplo, cuando se habla sobre la gastronomía chilena, los estudiantes extranjeros hablan sobre sus platos típicos. Estas comparaciones están acotadas a temas específicos, subrayando las diferencias más que las similitudes entre los distintos grupos culturales.

De las comunidades migrantes, la que más llama la atención por sus costumbres culturales es la de los estudiantes ecuatorianos, específicamente los otavaleños. Las familias de estos niños y niñas se caracterizan por conservar su identidad cultural, aun viviendo en Chile. Hablan en su lengua (Quichua), usan sus vestimentas y accesorios típicos. Especialmente las mujeres, que cotidianamente visten con los atuendos y joyas tradicionales.

Desde su experiencia profesional, los docentes identifican algunas cualidades o aspectos positivos que poseen los distintos grupos culturales. Los estudiantes peruanos y colombianos sobresalen por sus habilidades en comunicación oral. De los ecuatorianos no se enuncian cualidades cognitivas, pero sí se observa que poseen destrezas motrices para desarrollar trabajos manuales. Por otro lado, se observa que los estudiantes de estas nacionalidades muestran dificultades en la escritura. Los peruanos escriben lento y redactan respuestas muy breves. Los colombianos usan letra script y al cambiar a cursiva experimentan problemas con la grafía y el ligado de las letras. De hecho, algunos no se acostumbran a escribir con cursiva, a pesar de ejercitar caligrafía. Llama la atención que en ninguna de las entrevistas se haga mención a los estudiantes bolivianos, dominicanos y haitianos. No se señalan cualidades de su desempeño escolar ni se describen aspectos culturales. Quizás esto se deba a que son minoría en relación a las otras nacionalidades ya descritas.

Al momento de valorar las contribuciones o aportes culturales de los estudiantes migrantes, los profesores manifiestan que estas solo han sido de carácter personal, pero que no han enriquecido el desarrollo de las clases. Es decir, no son vistas como un recurso pedagógico relevante para producir aprendizajes y generar un diálogo intercultural. Esto llama la atención porque la mayoría de los profesores resaltan las habilidades en el ámbito lingüístico de peruanos y colombianos. Incluso comentan que los estudiantes chilenos, por medio de las interacciones con sus compañeros migrantes, han conocido nuevas palabras que no forman parte del vocabulario promedio del chileno. Esto les ha permitido comparar los distintos usos y significados que adquieren las palabras según el contexto sociocultural. 
Sin embargo, para los profesores esto no ha tenido una mayor repercusión en el aula, pues los chilenos no han mejorado el manejo y uso del español. Incluso, algunos dicen que después de un tiempo los extranjeros imitan la forma de hablar de sus compañeros chilenos. Por lo tanto, según los entrevistados no se produce un aprendizaje en el área del lenguaje.

A modo de explicación, para poder comprender esta paradoja, algunos docentes indican que los estudiantes extranjeros son retraídos y participan poco en clases. Esta actitud de ellos impediría un mayor reconocimiento y socialización de la diversidad cultural presente en el aula. Por otro lado, se dice que poseen un conocimiento limitado de la cultura de sus países. Pareciera ser que asimilan rápidamente la cultura chilena. Se observa que a muchos de ellos les cuesta reconocer su nacionalidad, especialmente a los estudiantes peruanos. En palabras de los entrevistados, esta pérdida de identidad se produce porque en la familia no se habla de sus países de origen ni se mantienen o fomentan sus tradiciones culturales.

Otro punto relevante en cuanto a la pérdida de la identidad nacional es que para los niños y niñas migrantes nacer en Chile se convierte en un hecho relevante que cambiará su condición de extranjeros. Para ellos, obtener la nacionalidad chilena es motivo de orgullo a pesar de que sus padres poseen otra nacionalidad. Esto se observa con frecuencia en los estudiantes peruanos, que hacen diferencias entre sus coterráneos que acceden a la nacionalidad chilena y los que conservan su nacionalidad de origen. Según los profesores, a los estudiantes peruanos que optan por la nacionalidad chilena les gusta ser reconocidos como chilenos, aunque muchos de ellos poseen padres y hermanos peruanos. Incluso comentan que por estos motivos se han producido peleas entre estudiantes peruanos y chileno-peruanos que tratan de ocultar su origen.

De todas las entrevistas, solo en dos casos se registran prejuicios y estereotipos que dan cuenta de una alteridad negativa. Las declaraciones de estas profesoras señalan que los estudiantes peruanos provienen de un país con un bajo nivel cultural. Que su sistema educacional es más atrasado porque varios de ellos llegan con vacíos pedagógicos. Afirman que son pocos los estudiantes peruanos que poseen las competencias para desenvolverse idóneamente en el curso que les corresponde. Además, señalan los estudiantes tienen bastantes problemas de aprendizaje, por lo que deben ser derivados a la educadora diferencial. De acuerdo a estos planteamientos, el fracaso escolar o las dificultades que estos niños y niñas experimentan se deben a un problema genético o al tipo de alimentación. También se reconoce que los estudiantes ecuatorianos presentan problemas de aprendizaje, eso sí en un número menor que los peruanos. Pero no se presentan comentarios negativos sobre ellos. De los estudiantes colombianos se comenta que son violentos, que están acostumbrados a resolver los conflictos por medio de los golpes o amenazas, al estilo de los sicarios. Porque muchos de ellos provienen de Cali u otras ciudades asociadas al narcotráfico.

Las opiniones que indican la presencia de estereotipos y prejuicios sobre algunas nacionalidades son reducidas. Aun cuando varios docentes sostienen implícitamente que los estudiantes peruanos son los que tienen más problemas y dificultades de aprendizaje. Esto revela que para varios docentes, Chile es superior a otros países de la región como Perú, Bolivia y Ecuador, la excepción es Colombia que muestra condiciones económicas y socioculturales similares a nuestro país.

La inclusión educativa de los estudiantes migrantes es lenta, a pesar de que se generan todas las condiciones para facilitar su adaptación a la escuela. Según los profesores, ellos llegan con una actitud hostil que dificulta su proceso de adaptación. Al principio son antisociales, violentos y reticentes a interactuar con sus pares. Sin embargo, luego de un tiempo logran 
integrarse a la escuela. La mayoría desarrolla buenas relaciones con sus compañeros de curso, y son muy pocos los casos de marginación. Los que han sido tratados por el psicólogo $\mathrm{u}$ orientadora del establecimiento obtienen buenos resultados. Las situaciones más complejas se han producido en los cursos superiores ( $5^{\circ}$ a $8^{\circ}$ año básico). Donde se han registrado más casos de discriminación, llegando incluso hasta las agresiones físicas.

Se desprende que tanto a nivel de discurso como en las observaciones, la mayoría de los docentes promueve la participación de todos los estudiantes durante el desarrollo de las clases. Atienden sus dudas y les entregan refuerzos positivos, independiente de su nacionalidad. Solo en dos cursos se observó que la participación de los estudiantes migrantes fue reducida. Varios de ellos no concluyeron las actividades de la clase y estuvieron bastante desconcentrados, a pesar de las ayudas entregadas por el docente.

En general se señala que no hay discriminación hacia los niños y niñas migrantes. Tampoco durante las observaciones se presenció un acto de violencia física, verbal o discriminación. Los casos que se conocen son puntuales. Ocurren a escondidas de los profesores o se generan fuera del aula. Los motivos por los que se discrimina, tienen relación con los rasgos físicos y la nacionalidad. Algunos docentes comentan que los colombianos han sido discriminados por tener la piel oscura y el pelo rizado, prueba de esto es que en una de las clases observadas una niña colombiana con rasgos afrodescendientes comentó que sus compañeros la molestaban con frecuencia por sus rasgos físicos. Mientras a los estudiantes peruanos se les discrimina por diversos motivos que aluden a la nacionalidad; es el grupo que más sufre burlas y malos tratos. En ese sentido, se constata que en las escuelas se estarían utilizando descalificativos específicos para denostar a los estudiantes de esa nacionalidad. Como prueba de esto, una profesora revela que a ellos les molesta mucho que les digan "come palomas". Igualmente, en otro establecimiento que no es parte de la investigación, uno de los profesores comentó el mismo hecho.

La nacionalidad se transforma en un elemento relevante entre los estudiantes al momento de establecer relaciones sociales. Es utilizada para diferenciar o identificarse con un determinado grupo. Se pudo observar que fuera de la sala de clases los estudiantes chilenos omiten los nombres de sus pares migrantes y los llaman por su nacionalidad. Antes de comenzar una de las observaciones, mientras los estudiantes esperaban formados para ingresar a la sala de clases, se registró el siguiente suceso: "Un niño le dice al que viene llegando atrasado llegó un colombiano, llegó un colombiano...me equivoqué era un ecuatoriano" (Obs., $3^{\circ}$ básico). En los cursos inferiores (primero y segundo básico) se observó que los niños y niñas no se refieren a sus compañeros por la nacionalidad, a diferencia de los niveles superiores. Pues al solicitarles a estudiantes de estos cursos que indicarán a sus compañeros extranjeros, solo pudieron reconocer a algunos y con bastante dificultad.

Las diferencias culinarias en la preparación de las comidas han causado que varios estudiantes migrantes no quieran comer de los alimentos que se les ofrecen en la escuela. Esto se debe específicamente a los aliños y condimentos utilizados. Para ellos las comidas que se les da son desabridas. Para remediar esta situación algunas mamas optaron por enviarles a sus hijos los aliños y condimentos para que sean añadidos a los platos de comida. En cambio, otros decidieron prepararles sus propios almuerzos. De esta forma, se logró que los estudiantes migrantes almuercen en la escuela y pudieran completar la jornada escolar sin contratiempos.

Otro dato que llama la atención es cómo un suceso externo puede repercutir en las relaciones entre los estudiantes. Dos profesores señalan que cuando juega la selección 
chilena de fútbol contra Perú, Ecuador o Colombia, se han producido discusiones o peleas que persisten en el tiempo, pero que no es algo generalizado. Estas rivalidades muestran cómo la nacionalidad es un elemento simbólico que genera conflictos. Por otra parte, la normativa interna de un establecimiento también puede provocar choques culturales. En una de las escuelas se recuerda que antes los estudiantes otavaleños llegaban con su cabello largo peinado en trenza. Como no cumplían con el reglamento, el director les exigió que tenían que cortarse el pelo como el resto de los estudiantes. Después de un tiempo se adaptaron para evitar problemas: ahora ellos asisten a clases con el pelo corto. Para los profesores que trabajaron en ese periodo, esta medida transgredía una costumbre tradicional de su pueblo.

En relación al espacio físico, dedicado para mostrar información de otras culturas y la distribución de los estudiantes extranjeros en el aula. Algunos profesores comentan que cuando recién comenzó el fenómeno migratorio implementaron un rincón o diario mural para exhibir información relacionada con Perú, ya que fue la primera comunidad extranjera que llegó al país. En esos espacios físicos se disponían los emblemas patrios, fotografías de lugares representativos, recetas y noticias. Además de esta experiencia, se menciona la elaboración de un glosario que contenía las palabras o modismos desconocidos para los estudiantes chilenos y peruanos. Sin embargo, las observaciones muestran que en ninguna de las aulas hay un lugar físico, como un diario mural, donde se muestren datos o imágenes relativas a las otras culturas, ni tampoco se ambientan las salas para mostrar la multiculturalidad, por ejemplo, con las banderas u otros símbolos. Solo en un primero básico de uno de los establecimientos había información alusiva a los otros países, este era el material de apoyo ocupado en una actividad denominada "Mi país", que se realiza anualmente y su objetivo es que los niños y niñas expongan sobre las tradiciones y costumbres de sus países.

Respecto a la distribución de los estudiantes al interior del aula, los docentes declaran que se relacionan e integran independientemente de la nacionalidad para realizar las actividades escolares. Una de las profesoras asegura que cuando se realizan trabajos grupales, a veces se juntan solo entre coterráneos, pero en esos casos el profesor debe intervenir para que todos los grupos sean diversos, y de esa forma se den las instancias formales para conocerse y respetarse. Sin embargo, durante las observaciones se evidencia que en varios cursos los migrantes están sentados entre sí, no comparten puestos con sus pares chilenos. Sobresale que en una de las salas una columna estaba conformada íntegramente por chilenos. En otros cursos los estudiantes migrantes están combinados con los chilenos y, en algunos puestos, se sientan según la nacionalidad. A diferencia de los casos anteriores, donde los niños y niñas escogen por afinidad con quien sentarse, existe un caso en que la distribución de los estudiantes es homogénea, en todos los puestos hay un chileno y un migrante. Esta regularidad demuestra que la profesora designa los puestos para favorecer la integración de los extranjeros.

4.2. DESARROLLO CURRICULAR: LAS TENSIONES Y POSIBILIDADES DEL CURRÍCULO NACIONAL

Las modificaciones que experimenta el currículum por medio del diálogo con el contexto escolar son mínimas. La implementación que se hace del currículum oficial se caracteriza por ser lineal: 
Yo sigo el currículo normal no más, el que da el ministerio de Chile $\left(\mathrm{E} 1,4^{\circ}\right)$.

en cuanto al currículo, no he tenido problema, no sé qué cambiaría en realidad, yo creo que $\operatorname{nada}\left(\mathrm{E} 4,2^{\circ}\right)$.

Los cambios que realizan los docentes en el currículo oficial son superficiales y se concentran en actividades puntuales. El modo más habitual es incorporar un dato o información de los otros países, específicamente, cuando el contenido lo permite:

Estamos viendo, por ejemplo, las leyendas, a ver ¿hay algún niñito que me quiera contar una leyenda de su país? ¿qué sé yo? salen cosas lindas que compartimos entre todos $\left(\mathrm{E} 4,2^{\circ}\right)$.

Estos actos de contextualización, en la mayoría de los casos, surgen de manera espontánea durante el desarrollo de las clases. Son generados por la intencionalidad del docente o producto de las intervenciones realizadas por los estudiantes migrantes. Los profesores afirman que constantemente están contextualizando el currículum. Pues dentro de la misma clase se van incorporando las experiencias de los estudiantes extranjeros. Sin embargo, no se evidencia el diseño de actividades para abordar la diversidad cultural. Una de las razones que explican la ausencia de actividades dirigidas es la sobrecarga de trabajo que poseen los docentes y las pocas horas destinadas para planificar. Además señalan que no se justifica hacer grandes modificaciones al currículum porque los estudiantes migrantes se adaptan rápidamente; y como se dijo anteriormente, esto puede resolverse de manera emergente durante el desarrollo de las clases.

Al momento de preguntar a los profesores por los contenidos más complejos para integrar aspectos culturales de los estudiantes migrantes, varios interpretaron que se les estaba consultando por las asignaturas donde estos niños y niñas experimentan más dificultades de aprendizaje. De acuerdo a los entrevistados las diferencias culturales representan dificultades en el ámbito del lenguaje y en la asignatura de Historia, geografía y ciencias sociales. En el desarrollo de las clases se han producido algunos problemas de comunicación por las variantes en la forma de hablar el español, según zona geográfica:

(...) recuerdo que un niño boliviano cuando tuvo que nombrar objetos que tuvieran forma de cono dijo el churro... Sus compañeros le dijeron que eso estaba mal y yo le pregunte ¿a qué se refería? y entonces el me explicó que en su país a los gorros de cumpleaños les decían churros $(\mathrm{E} 5,2)$.

Aunque estas variedades lingüísticas existen, los profesores señalan que son iniciales, porque después de un tiempo de permanencia los estudiantes conocen los modismos, significados y usos de las palabras. En este punto agregan que los estudiantes chilenos corrigen a sus pares extranjeros cuando utilizan ciertos términos que no son de uso corriente en Chile. Como el jalar en vez de tirar o el acabé por terminé.

Independiente de estos matices lingüísticos, en la actualidad no existen grandes problemas de comunicación al interior del aula, porque los distintos actores ya han identificado las palabras que generaban confusiones o impedían la decodificación de ciertos mensajes. Algunos de estos términos que forman parte del nuevo vocabulario escolar son: tajador (sacapuntas), mandil (delantal), tablero (pizarra), borrador (goma), etc. Tomando en consideración estas nuevas palabras, que se estaban usando cotidianamente en clases, 
uno de los profesores creó un glosario para facilitar la comunicación y mostrar la riqueza del lenguaje.

Sin embargo, hace seis u ocho años atrás se registraron problemas de comunicación producto de las diferencias lingüísticas. Los testimonios dan cuenta de las dificultades que tenían en el aprendizaje del español los estudiantes ecuatorianos, específicamente los otavaleños, por hablar en su lengua ancestral: el quichua. Esto generó en los padres bastante preocupación, porque no podían apoyar a sus hijos en los estudios. Aunque la barrera idiomática tuvo consecuencias académicas, en las primeras generaciones, en la actualidad los estudiantes otavaleños se adaptan con mayor rapidez que antes, aprendiendo con facilidad el español, sobre todo los niños y las niñas más pequeños.

En el aprendizaje de la lectoescritura también se originan confusiones por el uso de ciertas palabras. Una de las profesoras de primero básico comenta que cuando se enseñan las letras se debe tener cuidado con los ejemplos que se dan. Es común que en Chile se utilice la palabra e imagen de un auto para iniciar el estudio de la letra A. Pero para muchos estudiantes migrantes este ejemplo no es significativo, porque ellos le llaman a los autos “carros". Los docentes han podido solucionar estas diferencias lingüísticas a través de la experiencia profesional, identificando así los términos más adecuados y que sean de uso común, tanto para estudiantes migrantes como chilenos.

Las distintas metodologías empleadas por los sistemas educativos han producido desacomodos en la escolarización de los estudiantes migrantes. De acuerdo a los entrevistados, los métodos utilizados por países vecinos en lectoescritura ignoran la enseñanza de los fonemas de las letras. Esto quiere decir que durante este proceso no les enseñan los sonidos de los signos, como tradicionalmente se hace en Chile, sino que se centran en su forma pictórica o grafema, pasando de inmediato a la enseñanza del leguaje escrito:

Allá les enseñan la letra no más, no les enseñan que la letra " $M$ " su sonido es mmm. Entonces ellos me dicen tía, pero a mí nunca me pasaron el sonido de la letra. A ellos les dijeron solo el nombre de la letra, entonces los papás no saben enseñárselo a su hijo $\left(\mathrm{E} 2,1^{\circ}\right)$.

En ese sentido, los colombianos enfrentan problemas en la escritura, ya que en su país se enseña la escritura con letra script, a diferencia de Chile donde las bases curriculares establecen que todos los estudiantes deben escribir con cursiva. A nivel de asignaturas, en Historia, geografía y ciencias sociales se suscitan tensiones interculturales más evidentes, porque en su red de contenidos predominan temáticas nacionales y locales que son desconocidas para gran parte de los estudiantes migrantes, y además aparece el estudio de la Guerra del Pacífico, que continua pesando hasta hoy en las relaciones entre chilenos, peruanos y bolivianos.

Uno de los contenidos complejos para integrar aspectos culturales de los estudiantes migrantes es la geografía de Chile. A los niños y niñas extranjeros les cuesta ubicarse geográficamente en el país, específicamente a los que provienen de Ecuador y Colombia. También se dice que la mayoría poseen pocos conocimientos sobre sus países de origen. De acuerdo a los profesores desconocen aspectos elementales, como las capitales, héroes nacionales, las formas del relieve, los tipos de paisaje, el nombre del presidente de turno, etc., muchos de ellos han aprendido sobre sus países en Chile, mediante trabajos de investigación. Esta misma idea es compartida por los profesores, de dos establecimientos de la comuna de Santiago, donde se implementa el programa de Historia Suramericana. En 
su opinión "no es extraño que los estudiantes migrantes tengan problemas para aprender sobre un país desconocido, sino conocen ni el suyo”. Bajo ese planteamiento, algunos profesores argumentan que no es responsabilidad de ellos dicha situación, y que lo más adecuado es trabajar en base al currículo nacional, pues si ellos están en Chile, aunque sea de paso, deben aprender de nuestro país.

En cambio, para otros profesores, en geografía se producen dificultades iniciales, pero que al apoyarse con material concreto y audiovisual son superadas. Aunque el uso de estos recursos materiales y didácticos favorece el logro de aprendizajes en geografía, se observa que las diferencias del clima y relieve no son utilizadas como recursos valiosos para establecer comparaciones entre los países, teniendo en consideración que a través de las similitudes y diferencias es posible comprender el conjunto del territorio que comprende América del Sur. A modo de ejemplo, se podrían formular actividades donde se reconozca que la Cordillera de Los Andes es un elemento común para la mayoría de las nacionalidades presentes en el aula. (Ecuador, Colombia, Perú, Bolivia y Chile), o que en esos países no existen campos de hielo, así como en Chile no hay selva tropical, etc. Incluso se podría articular contenidos de Ciencias Naturales para ver cómo la ubicación geográfica influye en los recursos naturales que posee cada país.

La conmemoración del Combate Naval de Iquique es una fecha polémica que ha generado conflictos entre estudiantes peruanos y chilenos. Estas situaciones se han producido, principalmente desde $5^{\circ}$ a $8^{\circ}$ básico, pues en los cursos inferiores pareciera ser que por la corta edad de los niños y niñas, el concepto de nacionalidad todavía no está tan arraigado. Como una forma de aminorar estas hostilidades, los profesores más antiguos de la escuela República de Colombia recuerdan que en años anteriores, durante el 21 de mayo, se recreaba el combate Naval de Iquique, en esa oportunidad participaban estudiantes peruanos y chilenos. Otra estrategia utilizada para mediar en el conflicto es exponer los argumentos de ambas visiones históricas, destacando más que los hechos bélicos, las acciones simbólicas y admirables de los héroes, como las cartas de Grau y el heroísmo de Prat.

Estos antecedentes revelan que el punto álgido del programa de estudios de Historia, geografía y ciencias sociales radica en el origen de la nacionalidad chilena, la que se consolidó una vez concluida la Guerra del Pacífico, construyéndose una percepción negativa del otro, quien ha sido históricamente señalado como inferior. Una forma de superar esta visión sesgada es integrar los distintos saberes culturales de los estudiantes migrantes, abordando los contenidos desde una visión latinoamericana. Aunque la nacionalidad sigue siendo un imperativo estructurante de una alteridad negativa, existen hechos que demuestran que en las escuelas se están produciendo fuertes procesos de asimilación cultural o sincretismos culturales, al margen de las relaciones históricas con Perú y Bolivia. En ese sentido, pareciera ser que los imaginarios sociales siguen vigentes, no tanto por la conmemoración de fechas históricas, sino más bien por la influencia de los medios de comunicación y los continuos impases en la política exterior de los gobiernos. Por ejemplo, la contingencia noticiosa con la demanda de Perú ante la Haya produjo varios conflictos entre estudiantes chilenos y peruanos.

Existen contrastes entre los currículos escolares de la región, específicamente en la secuenciación de los contenidos por nivel educativo: de acuerdo a los profesores, el currículo nacional es más avanzado que el de otros países.

En relación a la procedencia de los estudiantes migrantes, se percibe que los peruanos llegan con un bajo nivel educativo. Muchos de ellos no poseen los conocimientos de base 
para abordar los contenidos que corresponden en los respectivos niveles de enseñanza. Por lo tanto, algunos creen que la contextualización curricular debe estar enfocada en la nivelación de contenidos, más que en aspectos culturales, ya que el fracaso escolar de estos estudiantes, tendría directa relación con el desfase en contenidos y capacidades exigidas por nivel. En cambio, otros profesores reconocen que a los estudiantes peruanos les cuesta adaptarse en el sistema educativo chileno, al igual que al resto de los estudiantes. Pero una vez que lo hacen, sobresalen académicamente en sus respectivos cursos. Si bien hay diferencias en la secuenciación de contenidos, hay un desconocimiento absoluto de la organización curricular de los países vecinos, lo que podría estar influyendo en la valoración de sus sistemas educativos.

A nivel de aula, la implementación de actividades interculturales es reducida y acotada a ciertas asignaturas. Para los docentes, estas actividades poseen un carácter transversal, aunque se puede constatar que se generan, principalmente, en las asignaturas de Historia, geografía y ciencias sociales, Lenguaje y comunicación y Educación artística. Esto se debe a la conexión y facilidad que otorgan los contenidos declarados en sus programas de estudio para incluir datos o informaciones de los países de origen de los estudiantes migrantes.

En Historia, geografía y ciencias sociales, los temas empleados comúnmente para estos fines son: los emblemas patrios, las comidas, vestimentas y bailes típicos. En menor grado, se incluyen lugares simbólicos como las ruinas de Machu Picchu o los tipos de paisajes. En Lenguaje y comunicación se identifican como temas integradores los mitos y leyendas y el vocabulario que emplean los estudiantes migrantes. Para el caso de Educación artística, los contenidos de esta asignatura son utilizados para crear o expresar productos culturales de las diversas nacionalidades, por ejemplo la elaboración de banderas, collages con imágenes representativas de sus países y jarros de greda con decoraciones o motivos típicos. Sin embargo, las observaciones indican que el desarrollo de los contenidos no se adapta ni articula a la realidad sociocultural de los estudiantes migrantes. Teniendo en consideración que los contenidos de las asignaturas de Lenguaje y comunicación o Historia, geografía y ciencias sociales facilitan el diálogo intercultural. La mayoría de las observaciones se realizó en las horas correspondientes a estas asignaturas, pero quedó al descubierto que no es prioridad integrar los saberes o conocimientos provenientes de otras culturas, tal como se puede percibir en la siguiente nota de campo, registrada durante una clase de un tercero básico en la asignatura de Historia, geografía y ciencias sociales, mientras se enseñaba la Unidad Representaciones de la Tierra:

Los estudiantes identifican en un mapamundi las coordenadas geográficas, los continentes y océanos. Luego la profesora pregunta, entre otras cosas ¿en qué continente se ubica Chile? ¿cuál es la línea imaginaria que divide la Tierra en dos hemisferios? En ningún momento de la clase se consideraron los países de origen de los niños migrantes. Solo se preguntó por la ubicación geográfica de Chile. A pesar de que habían varios estudiantes ecuatorianos, se desestimaron los conocimientos que ellos podrían tener sobre la línea del Ecuador (Obs. $n^{\circ}$ 8).

La implementación de actividades curriculares planificadas y que den cuenta de una lectura profunda del marco curricular son inexistentes. Tampoco se incorporan temas nuevos ni se elaboran guías de aprendizaje que reconozcan la multiculturalidad que existe en el aula; solo en dos clases los estudiantes migrantes intervinieron y difundieron datos de sus países de origen; solo en uno de los establecimientos se desarrolla una actividad 
dirigida especialmente a los estudiantes migrantes, esta consiste en que cada estudiante debe preparar una disertación de su país de origen, mostrando a sus compañeros los aspectos más representativos de cada cultura nacional. En las disertaciones participan los familiares de los estudiantes y finalizan con una demostración de una canción, bailes o comida típica. Esta actividad de integración es anual y se realiza en primero y segundo básico. Aparte de esta actividad que se desarrolla exclusivamente por la inquietud de las profesoras de primero básico, llama la atención que ninguno de los profesores diseñe e implemente otras acciones, para fomentar un diálogo intercultural entre los distintos grupos que conviven en el aula.

En síntesis, las actividades descritas, en la mayoría de los casos, surgen de manera espontánea durante el desarrollo de las clases. Generalmente, los temas generadores con los cuales se intenta promover un diálogo intercultural tienen relación con los emblemas patrios, comidas, bailes típicos, leyendas, patrimonios culturales y vocabulario. Sin embargo, llama la atención que no se realicen actividades para analizar los imaginarios sociales surgidos por la Guerra del Pacífico cuando los mismos profesores reconocen que el concepto de nacionalidad genera odiosidades entre los estudiantes chilenos y peruanos, reproduciéndose un discurso identitario de superioridad, cimentado por el triunfo de la guerra, que impide un reconocimiento del otro como un ser igual.

Al consultar a los profesores si la diversidad cultural está contemplada por el marco curricular, las opiniones se muestran divididas. Para un grupo la atención a la diversidad es un tema prioritario que ha ido en desarrollo durante los últimos diez años. En palabras de estos profesores los lineamientos de la política educativa en este tema incluyen las distintas expresiones del concepto, como las necesidades educativas especiales, la educación de los pueblos originarios y de los discapacitados. Por lo tanto, las sugerencias necesarias para afrontar las diferencias culturales existen, y no sería necesario hacer un apartado especial sobre los migrantes. Este grupo de educadores percibe que con la implementación de las bases curriculares del año 2012 se produjeron cambios notorios en los programas de estudio, pudiéndose observar que en los actuales libros de texto del estudiante se incorporaron más temas relacionados con las distintas culturas.

De acuerdo a estos planteamientos, la diversidad cultural ocupa un espacio importante en el texto de Historia, geografía y ciencias sociales. Se denota un énfasis en contenidos, que antes no se desarrollaban con tanta profundidad, como las grandes civilizaciones de Latinoamérica y actividades para trabajar la tolerancia y el respeto de todas las culturas. En el texto de Lenguaje y comunicación, como consecuencia de los ajustes curriculares, se incluye una vasta recopilación de literatura latinoamericana. Al contrario de la valoración positiva que se hace de los textos de estudio por incorporar la diversidad como un eje temático transversal en la organización de los contenidos, una de las profesoras cuestiona la homogeneidad cultural de la asignatura de Historia, geografía y ciencias sociales. También se hace un llamado a las autoridades del MINEDUC por la falta de recursos o materiales curriculares, que faciliten el trabajo de los docentes que se desempeñan en establecimientos con estudiantes migrantes.

La mayoría de los profesores utiliza, preferentemente, el texto de estudio entregado por el Ministerio de Educación, además de recursos complementarios como guías, videos, material concreto y didáctico. No se percibe la elaboración de materiales curriculares con la finalidad de integrar los saberes culturales de los estudiantes migrantes, solo se hace mención del uso de recursos audiovisuales para mostrar algún baile o animación de alguna leyenda extranjera. 
Tomando en consideración la innovación intercultural llevada a cabo en la Escuela República de Alemania, que dio origen a un plan y programa de estudio propio para séptimo básico, denominado "Historia, geografía y ciencias sociales de Suramerica", se les consultó a los profesores por la pertinencia de crear programas o textos de estudio para poder trabajar con los estudiantes migrantes. En opinión de algunos profesores, la elaboración de un programa o texto de estudio especial para la integración de las distintas culturas no es necesaria ni se justifica. Primero porque los estudiantes migrantes se adaptan rápidamente a la cultura chilena y no tienen inconvenientes con los textos de estudios. Segundo, presentan las mismas dificultades que los estudiantes chilenos, por lo que no es necesario disponer de un texto de apoyo especial. Tercero, la diversidad cultural de América Latina ya está contemplada por el marco curricular oficial. En ese sentido, incluir más datos o informaciones sería un exceso de contenidos. Por último, deben aprender y conocer la cultura de Chile, porque es el país donde viven.

Desde otro punto de vista, en una de las entrevistas se señaló que implementar un programa de estudio dirigido a los niños y niñas migrantes podría convertirse en una forma de discriminación. Sin embargo, es importante aclarar que la innovación curricular responde al contexto multicultural, pero su implementación está dirigida a todos los estudiantes, pues el objetivo es promover un diálogo entre todos los actores con el objetivo de respetar y valorar la diversidad cultural existente en la escuela, tal como ocurre en la Escuela República de Alemania, donde el programa de Historia Suramericana se transformó en una asignatura para estudiantes chilenos y extranjeros que cursan séptimo básico. Otro argumento interesante para negar la creación de un programa de estudio que responda a la migración es la reivindicación que se hace de las culturas originarias de Chile ante otras culturas foráneas, pues estas tendrían mayor legitimidad por pertenecer a la historia de nuestro país, y hasta ahora tampoco han sido reconocidas por el currículum oficial. Por lo tanto, en vez de crear textos para los migrantes, deberían elaborarse textos, por ejemplo, para enseñar mapudungun.

En cambio, otros profesores aprueban la idea de realizar cambios curriculares en favor de los estudiantes migrantes. Al interior de este grupo es posible identificar dos visiones: algunos creen que es necesario generar propuestas concretas para atender a estos niños y niñas, más allá de las contextualizaciones del currículo que se puedan hacer en el aula; otros consideran que se deberían agregar a los programas de estudio existentes unidades o contenidos transversales relacionados con las distintas culturas.

Frente a estas opiniones en pro o en contra de un programa de estudio especial que aborde la migración, la escuela no puede ignorar los cambios que ha experimentado la sociedad chilena durante este último tiempo. Por lo tanto, sería pertinente producir materiales de apoyo necesarios para fortalecer su educación, teniendo en consideración que la matrícula de estudiantes migrantes va en aumento y que la mayoría ingresa a establecimientos municipales.

\section{CONCLUSIONES}

Los resultados obtenidos por esta investigación permiten concluir que los profesores en su quehacer profesional promueven la participación de todos los estudiantes al interior del aula, independiente de su nacionalidad. Bajo este principio de igualdad educativa, se 
otorgan las mismas oportunidades de aprendizaje para todos los niños y niñas; también se generan intercambios culturales entre los distintos grupos. En las clases, los estudiantes migrantes pueden difundir aspectos culturales de sus países, cuando esto sucede los estudiantes chilenos manifiestan curiosidad y respeto por los saberes de los estudiantes migrantes. Aunque existen estos espacios de diálogo entre las otras culturas, se observa que no es prioridad integrar la diferencia cultural como un recurso que puede enriquecer los aprendizajes y la implementación del currículum.

Las escuelas en contextos de alumnado migrante no han logrado implementar una política inclusiva que facilite la integración de los niños y niñas extranjeros. Se evidencia la ausencia de un plan y actividades concretas para eliminar la discriminación, el racismo y la xenofobia. En este contexto, el respeto y la tolerancia con la diversidad cultural es desarrollado, principalmente, por la iniciativa personal de cada docente. Si bien al interior del aula lograron disminuirse las conductas discriminatorias y racistas hacia los estudiantes migrantes, la evidencia indica que ahora estas suceden fuera del aula en espacios informales, donde la supervisión de los adultos es menor, tales como patio, baños o comedores de la escuela.

Los motivos por los que discriminan los estudiantes chilenos tienen relación con los rasgos físicos y la nacionalidad. Los grupos culturales que frecuentemente reciben tratos vejatorios y ofensivos son los estudiantes peruanos y afrocolombianos. Es importante advertir que de todas las nacionalidades mencionadas, los más discriminados son los peruanos. Esto es preocupante considerando que en los establecimientos municipales con alta presencia de alumnado migrante, la mayor cantidad de matrícula corresponde a este grupo.

La adaptación de los estudiantes migrantes posee las características de una asimilación cultural forzosa más que dialógica. De acuerdo a los profesores, su adaptación es lenta porque ellos manifiestan, al comienzo, una actitud hostil que dificulta la interacción con sus pares. Este sentir es parte del imaginario social y delimita las acciones o condiciones que se puedan generar para facilitar el proceso de integración. Bajo esa premisa la responsabilidad siempre recae en el otro, pues al ser ellos los extranjeros, son ellos los que deben integrarse. En este contexto, los casos más complejos de exclusión se han producido desde quinto hasta octavo año básico.

En el ámbito de las representaciones sociales que se han ido construyendo en las relaciones intersubjetivas del proceso educativo, el concepto de nacionalidad es el elemento simbólico que más impacto produce al interior de las escuelas. Para los estudiantes es un aspecto relevante al momento de establecer relaciones sociales, utilizada para diferenciar o identificarse con un determinado grupo. Por otro lado, es una fuente de conflictos con los estudiantes peruanos en fechas conmemorativas como las Glorias navales. En el caso de los docentes, se observa que estos construyen la percepción del otro de acuerdo a la nacionalidad. Aunque la presencia explícita de prejuicios y estereotipos fue reducida, se observa que existe una subvaloración del desempeño escolar de los estudiantes peruanos.

La diversidad producida por la migración no es valorada por los educadores como un aporte significativo para la clase. Aunque se reconocen cualidades de las otras culturas, como el desempeño lingüístico de peruanos y colombianos o las costumbres de los otavaleños, los profesores manifiestan que estas solo han sido de carácter personal, pero que no han enriquecido el desarrollo de las clases ni han contribuido a la mejora de los aprendizajes. 
El currículum que se implementa en las escuelas con estudiantes migrantes no experimenta grandes modificaciones ni interpretaciones. La contextualización curricular se reduce a la inserción de datos o informaciones aisladas de las otras culturas, sin mayores reestructuraciones del mismo. Estos actos de contextualización, en la mayoría de los casos, surgen de manera espontánea, quedando en evidencia la ausencia de un proceso de planificación para poder articular los contenidos declarados en el marco curricular con los saberes culturales de los estudiantes migrantes.

Las orientaciones curriculares y metodologías empleadas por cada sistema educativo deben ser contempladas en la educación de los niños y niñas migrantes. Los datos indican que en los países vecinos se implementan otros métodos en lectoescritura, diferencias han producido desacomodos en el desempeño escolar de los estudiantes. En ese sentido, sería interesante que a nivel ministerial se realice una comparación entre los distintos currículos de la región (de los principales flujos migratorios que llegan al país) para lograr una articulación curricular y así conocer con exactitud los programas y aprendizajes que poseen los estudiantes migrantes. De esta forma se facilitaría el reconocimiento de los certificados y la incorporación.

Las asignaturas más favorables para la integración de las otras culturas son Lenguaje y comunicación, Historia, geografía y ciencias sociales y Educación artística. Por lo tanto, estas áreas disciplinares debieran considerarse al momento de elaborar proyectos de innovación intercultural. Generalmente, los temas generadores con los cuales se intenta promover un diálogo tienen relación con los emblemas patrios, comidas, bailes típicos, leyendas, patrimonios culturales y vocabulario.

El desarrollo de actividades para reconocer la diversidad cultural es escaso y está circunscrito a fechas conmemorativas. Principalmente, en las escuelas se realizan actos o ferias escolares donde se brindan espacios para que los estudiantes migrantes puedan mostrar costumbres de sus respectivos países. Sin embargo, a nivel de aula solo en uno de los establecimientos se realiza una actividad periódica. Esta consiste en una disertación de los aspectos representativos de cada país y finaliza con una degustación de comida típica o la presentación de un baile.

La Guerra del Pacífico es el nudo crítico del programa de Historia, geografía y ciencias sociales. Las representaciones sociales originadas por el conflicto todavía se mantienen y generan odiosidades entre estudiantes chilenos y peruanos. Como estrategia para aminorar las tensiones, los profesores han incorporado paulatinamente las dos visiones históricas del conflicto. Aunque esto es un gran avance, es imprescindible promover acciones en la enseñanza de la historia para hacer visibles y eliminar la transmisión de prejuicios xenófobos y racistas. En paralelo al conflicto, es necesario buscar otros temas que demuestren que las relaciones históricas de Chile y Perú no se limitan solo a la guerra, por ejemplo, se pueden destacar los lazos prehispánicos, las relaciones comerciales entre ambos países o los aspectos positivos de la migración peruana en Chile.

El estado en que se encuentra la educación de los niños y niñas migrantes es preocupante, porque revela que en las escuelas las actividades interculturales están abocadas solo a un día o a las efemérides del calendario escolar. Es decir, solo en esa ocasión se da cuenta de la multiculturalidad y el resto del año todo sigue normal. Esto demuestra que gran parte de los establecimientos con alumnado migrante no ha logrado implementar un enfoque intercultural que supere la etapa del reconocimiento para trascender a la interacción entre todas las culturas. Además, los antecedentes advierten que hay una falta de preparación de 
los docentes para afrontar el conflicto cultural, debido a una formación inicial homogénea y a la implementación de un currículo descontextualizado. Por otro lado, está la necesidad de contar con una institucionalidad sólida por parte del Ministerio de Educación y los municipios que fomente y apoye proyectos de innovación curricular.

\section{REFERENCIAS BIBLIOGRÁFICAS}

Aranda, V. (2011). Reflexión y análisis de políticas y prácticas innovadoras a la luz de las representaciones sociales y de la necesidad de una educación intercultural en la formación inicial docente. Estudios Pedagógicos, 37(2), 301-314.

Departamento de Asuntos Económicos y Sociales de las Naciones Unidas. (2013). Tendencias en el número de migrantes internacionales: Revisión año 2013. Recuperado desde: http://esa.un.org/ unmigration/TIMSO2013/migrantstocks2013.htm

Dirección de Bibliotecas, Archivos y Museos (DIBAM). Innovación curricular para colegios con alto alumnado migrante. Recuperado desde http://www.contenidoslocales.cl/comunidad/2851/ innovacion-curricular-para-colegios-con-alto-alumnado-inmigrante

Instituto Nacional de Estadísticas. (2003). Resultados XVII Censo de Población 2002, Síntesis de resultados. Santiago de Chile: Autor.

Instituto Nacional de Estadísticas. (2014). Resultados XVIII Censo de Población 2012, Tomo I. Santiago de Chile: Autor.

Martínez, J. (2003). El encanto de los datos. Sociodemografía de la inmigración en Chile según el censo de 2002. Serie Población y Desarrollo, 49. Santiago de Chile: CELADE-CEPAL. Publicación de las Naciones Unidas.

Ministerio Secretaría General de Gobierno. (2013). Primera Consulta Ciudadana sobre la Discriminación en Chile. Santiago de Chile: Autor.

Organización de los Estados Americanos. (2011). Educación para niñas, niños y jóvenes inmigrantes en las Américas: Situación actual y desafíos. Autor.

Pavez, I. (2013). Los significados de ser niña y niño migrante: conceptualizaciones desde la infancia peruana en Chile. Revista Polis, 35, 1-19. Centro de Investigación Sociedad y Políticas Públicas (CISPO). Recuperado desde: http://polis.revues.org/9304

Poblete, R. (2006). Educación intercultural: teorías, políticas y prácticas. La migración peruana en el Chile de hoy. Nuevos escenarios y desafíos para la integración (Tesis doctoral). Universidad Autónoma de Barcelona, España.

Portal de educación Educar Chile. (s.f.) Inmigración e interculturalidad: desafío de la escuela. Recuperado desde: http://www.educarchile.cl/Portal.Base/Web/VerContenido. aspx?ID=196588\#2

Soffia, M., Cano, M., \& Martínez, J. (2009). Conocer para legislar y hacer política: los desafíos de Chile ante un nuevo escenario migratorio. Serie Población y Desarrollo, 88. Santiago de Chile: CELADE-CEPAL. Publicación de las Naciones Unidas.

Stefoni, C. (2011). Perfil migratorio de Chile. Buenos Aires, Argentina: Publicación de la Organización Internacional para las Migraciones.

Tijoux, M. (2013). Las escuelas de la inmigración en la ciudad de Santiago: Elementos para una educación contra el racismo. Revista Polis, 35, 1-15. Centro de Investigación Sociedad y Políticas Públicas (CISPO). Recuperado desde: http://polis.revues.org/9338

Torres, C. (Coord.). (2011). Proyecto Ciudadanía y protección de los derechos humanos de la población inmigrante en Chile. Santiago de Chile: Fundación Instituto de la mujer.

UNICEF. (2004). Informe cuantitativo primera parte. Convivencia en el ámbito escolar. Santiago, Chile: Publicación de las Naciones Unidas. 
